\title{
Do dental calculi predict the presence of renal stones?
}

\author{
Bulent Kati ${ }^{1}$, Ergin Kalkan ${ }^{2}$, Eyyup Sabri Pelit ${ }^{1}$, Ismail Yagmur ${ }^{1}$, Halil Çiftçi ${ }^{1}$ \\ ${ }^{1}$ Department of Urology, Harran University Faculty of Medicine, Sanliurfa, Turkey; \\ ${ }^{2}$ Cagri Dental Hospital, Dental Clinic, Elazig, Turkey.
}

\begin{abstract}
Summary Objective: Pathological calcifications that occur in various parts of the body may cause stone formation over time. The structure of these stones is similar in many regions of the body. We have studied the relationship between dental calculi and kidney stones.

Material and methods: A total of 183 patients with dental stone complaints or dental calculi were included between April and August 2016 in the Cagri Dental Hospital, Elazig, Turkey. Patients were evaluated with regard to a urinary tract ultrasonography, urinalysis, oral hygiene, and stone and surgical disease history. All information was statistically investigated. Results: The age of the patients in the kidney stones group was significantly higher than the non-kidney stone patients $(p<0.05)$. In the group with kidney stones, the percentage of dental calculus formation was significantly higher than the group without stones $(p<0.05)$. In the groups with and without kidney stones, dental stone recurrence rates did not differ significantly $(p<0.05)$. Urinary $p H$ was significantly lower in the group with stones than the group without stones $(p<0.05)$. Conclusions: During a physical examination, the formation of a visible stone, such as a dental calculus, may be an indicator of other types of stones, such as kidney stones, and this should be further investigated.
\end{abstract}

KEY WORDS: Renal stone; Dental calculi; Stone formation; Etiology.

Submitted 16 May 2018; Accepted 5 July 2018

\section{INTRODUCTION}

In dentistry, a calculus or tartar is a form of hardened dental plaque. These are caused by the precipitation of minerals from saliva and gingival crevicular fluid in plaque on the teeth. This precipitation process kills the bacterial cells within the dental plaque, but the rough and hardened surface that is formed provides an ideal surface for further plaque formation. This leads to a calculus build up, which compromises the health of the gingiva. A calculus can form both along the gum line (supragingival) and within the narrow sulcus between the teeth and the gingiva (subgingival) (1).

Calculus formation is associated with a number of factors including sex, age, bad breath, receding gums, and chronically inflamed gingival (2). Brushing and flossing can remove the plaque from which a calculus forms. However, once formed, it is too hard and firmly attached to be removed with a toothbrush. Calculus buildup can be removed with ultrasonic tools or dental hand instru- ments (such as a periodontal scaler). Calculi are composed of both inorganic (mineral) and organic (cellular and extracellular matrix) components. The mineral portion of a calculus ranges from 40-60\%, depending on its location in the dentition, and consists primarily of calcium phosphate crystals organized into four principal mineral phases: octacalcium phosphate, hydroxyapatite, whitlockite, and brushite. The organic component of a calculus is approximately $85 \%$ cellular and 15\% extracellular matrix (3).

Renal stones, which are part of a multifactorial disease, are some of the most common problems in modern society and may affect $12-15 \%$ of the population with observation of an increase in their prevalence. Renal stones are more common in males and are categorized into calcareous (calcium containing) stones, which make up 90\% of all stones, and non-calcareous stones. Most stones (85\%) primarily contain calcium oxalate $(\mathrm{CaOx})$ admixed with calcium phosphate (CaP) in the form of apatite or brushite, or occasionally uric acid; however, less commonly they can be composed primarily of CaP. Although many systemic diseases, such as primary hyperparathyroidism, bowel disease, and renal tubular acidosis, can result in calcium stone formation, the majority of calcium stones are found in people with no systemic illness (4). Although many inherited and systemic diseases are associated with calcium renal stones, most stones are idiopathic (5).

In this study, we aimed to evaluate the presence of kidney stones in patients with dental calculus. This was performed according to the dental plaque density in patients who were treated at the same time in the urology clinic. We also aimed to uncover any relationships between these conditions.

\section{Materials AND METHOdS}

The study group was composed of 183 patients (86 females and 97 males) with an age range from 20 to 65 years. We randomly evaluated dental patients who were diagnosed with dental calculus. Based on the amount of plaque, patients were divided into three categories. For the purposes of X-ray assessments of the jaw, panoramic, and retroalveolar region, X-rays in suspicious teeth were made. For the realization of the set objective, visual analyses of the X rays were conducted (Figure 1).

Dental calculus amounts that are generally below 25\% 


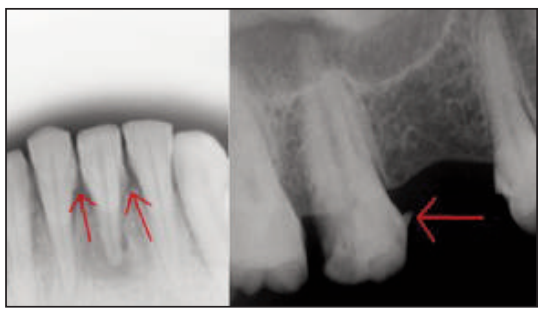

Figure 1.

$X$-ray views of dental calculi (arrows).

Figure 2a, $b, c$.

Appearance of dental calculus during a physical examination.

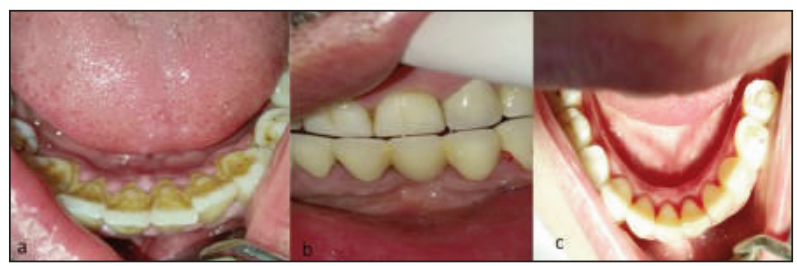

Figure 3.

The appearance of kidney stones and crystals in the kidney ultrasound (arrows).

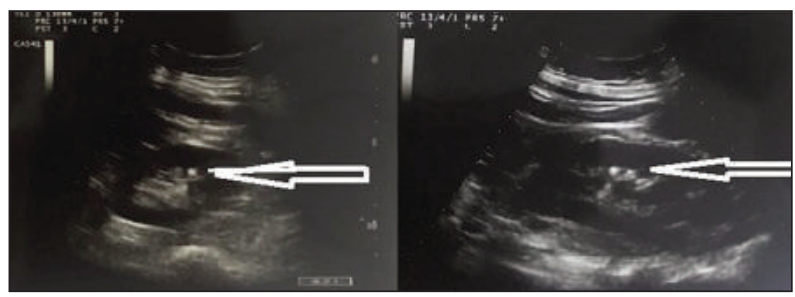

are termed as "low category," tartar amounts from 25$75 \%$ as "medium category," and as "high category" those amounts between $75-100 \%$ (Figure 2).

After the dental examination, all patients were evaluated with a urinary tract ultrasound and urinalysis (Figure 3). Fasting second morning specimens were collected from all individuals who had been fasting since 9:00 p.m. the preceding evening. The dental brushing habits of the patients were evaluated in terms of oral hygiene, additional disease, and stone story. All information was collected to determine the incidence of dental calculus and kidney stone in patients.

\section{Statistical analysis}

Mean, standard deviation, median lowest and highest, frequency, and ratio values were used in the descriptive statistics of the data. The distribution of the variables was measured by the Kolmogorov-Smirnov test. The MannWhitney $U$ test was used in the analysis of quantitative data. The chi-square test was used in the analysis of qualitative data. The SPSS 22.0 program was used for all statistical analyses.

\section{RESULTS}

The data from all patients were evaluated together (Table 1). The age of the patients in the kidney stone group was significantly higher than that of the non-kidney stone group $(p<0.05)$. The proportion of patients with renal stones who were males was significantly higher than the group without kidney stones $(p<0.05)$. The dental calculus formation was more prevalent in those without renal stones $(p<0.05)$. In the groups with and without kidney stones, dental stone recurrence rates did not differ significantly $(\mathrm{p}>0.05)$. Urinary $\mathrm{pH}$ was significantly lower in the group without stones $(\mathrm{p}<0.05)$ (Table 2).

In the univariate model, there was a significant difference $(p<0.05)$ in the age, sex, tooth stone percentage, tooth brushing habits, recurrence frequency, presence of addi-

\begin{tabular}{|c|c|c|c|c|c|}
\hline & & Min-Max & Median & \multicolumn{2}{|c|}{$\mathrm{t} \pm \mathrm{s} .5 . / \mathrm{n}-\%$} \\
\hline Age & & $18,0-91,0$ & 39,0 & \multicolumn{2}{|c|}{$41,4 \pm 16,5$} \\
\hline \multirow{2}{*}{ Sex } & Female & & & 86 & $47,0 \%$ \\
\hline & Male & & & 97 & $53,0 \%$ \\
\hline \multirow{3}{*}{ Dental calculi percent (\%) } & $0-25$ & & & 87 & $47,5 \%$ \\
\hline & $25-75$ & & & 62 & $33,9 \%$ \\
\hline & $75-100$ & & & 34 & $18,6 \%$ \\
\hline Recurrence (month) & & $4,0-48,0$ & 18,0 & \multicolumn{2}{|c|}{$18,9 \pm 11,0$} \\
\hline \multirow{2}{*}{ Brush teeth } & Regularly & & & 90 & $49,2 \%$ \\
\hline & Irregularly & & & 93 & $50,8 \%$ \\
\hline \multirow{2}{*}{ Comorbidities } & No & & & 123 & $67,2 \%$ \\
\hline & Yes & & & 60 & $32,8 \%$ \\
\hline \multirow{3}{*}{ Oral Hygiene } & Bad & & & 34 & $18,6 \%$ \\
\hline & Intermediate & & & 76 & $41,5 \%$ \\
\hline & Good & & & 73 & $39,9 \%$ \\
\hline \multirow{2}{*}{ Renal Stone History } & No & & & 152 & $83,1 \%$ \\
\hline & Yes & & & 31 & $16,9 \%$ \\
\hline \multirow{2}{*}{ Stone Surgery History } & Yes & & & 10 & $32,3 \%$ \\
\hline & No & & & 21 & $67,7 \%$ \\
\hline \multirow{2}{*}{\multicolumn{2}{|c|}{$\begin{array}{l}\text { Urine ph } \\
\text { Urine Dansity }\end{array}$}} & $5,0-7,0$ & 6,0 & \multicolumn{2}{|c|}{$5,8 \pm 0,5$} \\
\hline & & $1011-1030$ & 1021 & \multicolumn{2}{|c|}{$1020 \pm 6,1$} \\
\hline
\end{tabular}

Table 1.

Characteristics of patients being treated for dental calculi. 


\begin{tabular}{|c|c|c|c|c|c|c|c|c|}
\hline & & \multicolumn{3}{|c|}{ Stone - No } & \multicolumn{3}{|c|}{ Stone-Yes } & \multirow{2}{*}{$p$} \\
\hline & & \multicolumn{2}{|c|}{$\mathrm{t} \pm \mathrm{s.5.} / \mathrm{n}-\%$} & \multirow{2}{*}{$\frac{\text { Median }}{34,0}$} & \multicolumn{2}{|c|}{$\mathrm{t \pm s.s./n- \%}$} & Median & \\
\hline Age & & 37,8 & $\pm 15,6$ & & 52,0 & 14,6 & 53,0 & $0,000 \mathrm{~m}$ \\
\hline \multirow{2}{*}{ Sex } & Female & 70 & $52,6 \%$ & & 16 & $34,0 \%$ & & \multirow{2}{*}{$0,039^{x^{2}}$} \\
\hline & Male & 66 & $49,6 \%$ & & 31 & $66,0 \%$ & & \\
\hline \multirow{3}{*}{$\begin{array}{l}\text { Dental calculi } \\
\text { percent }(\%)\end{array}$} & $0-25$ & 70 & $52,6 \%$ & & 17 & $36,2 \%$ & & \multirow{3}{*}{0,002} \\
\hline & $25-75$ & 49 & $36,8 \%$ & & 13 & $27,7 \%$ & & \\
\hline & $75-100$ & 17 & $12,8 \%$ & & 17 & $36,2 \%$ & & \\
\hline \multicolumn{2}{|c|}{ Recurrence (Month) } & \multicolumn{2}{|c|}{$17,9 \pm 10,1$} & 16,5 & \multicolumn{2}{|c|}{$21,6 \pm 12,9$} & 18,0 & $0,134^{m}$ \\
\hline \multirow{2}{*}{ Brush Teeth } & Regularly & 59 & $44,4 \%$ & & 31 & $66,0 \%$ & & \multirow{2}{*}{$0,008^{x^{2}}$} \\
\hline & Irregularly & 77 & $57,9 \%$ & & 16 & $34,0 \%$ & & \\
\hline \multirow{2}{*}{ Comorbidities } & No & 98 & $73,7 \%$ & & 25 & $53,2 \%$ & & \multirow{2}{*}{$0,018^{x^{2}}$} \\
\hline & Yes & 38 & $28,6 \%$ & & 22 & $46,8 \%$ & & \\
\hline \multirow{3}{*}{ Oral Hygiene } & Bed & 14 & $10,5 \%$ & & 20 & $42,6 \%$ & & \multirow{3}{*}{0,000} \\
\hline & Intermediate & 62 & $46,6 \%$ & & 14 & $29,8 \%$ & & \\
\hline & Good & 60 & $45,1 \%$ & & 13 & $27,7 \%$ & & \\
\hline \multirow{2}{*}{ Stone History } & No & 132 & $99,2 \%$ & & 20 & $42,6 \%$ & & \multirow{2}{*}{$0,000^{x^{2}}$} \\
\hline & Yes & 4 & $3,0 \%$ & & 27 & $57,4 \%$ & & \\
\hline \multicolumn{2}{|c|}{ Stone Surgery History } & 1 & $25,0 \%$ & & 9 & $33,3 \%$ & & \\
\hline \multicolumn{2}{|l|}{ Urine ph } & \multicolumn{2}{|c|}{$5,7 \pm 0,5$} & 5,8 & \multicolumn{2}{|c|}{$5,9 \pm 0,5$} & 6,0 & $0,031 \mathrm{~m}$ \\
\hline \multicolumn{2}{|l|}{ Urine density } & \multicolumn{2}{|c|}{$1020 \pm 6,1$} & 1018 & \multicolumn{2}{|c|}{$1020 \pm 6,4$} & 1021 & $0,748^{m}$ \\
\hline
\end{tabular}

Table 2.

Statistical comparisons of patient

information.

\begin{tabular}{|c|c|c|c|c|c|c|}
\hline & \multicolumn{3}{|c|}{ Univariate Analysis } & \multicolumn{3}{|c|}{ Multivariate Analysis } \\
\hline & Mean & $95 \% \mathrm{GA}$ & $\mathrm{p}$ & Mean & $95 \% \mathrm{GA}$ & $p$ \\
\hline Age & 1,06 & $1,03-1,08$ & 0,000 & 1,03 & $1,00-1,06$ & 0,024 \\
\hline Sex & 2,05 & $1,03-4,10$ & 0,041 & & & \\
\hline Dental Calculi Percent & 3,97 & $1,81-8,67$ & 0,001 & 4,38 & $1,60-12,00$ & 0,004 \\
\hline Brush Teeth Habit & 0,40 & $0,20-0,79$ & 0,009 & & & \\
\hline Recurrence (month) & 1,03 & $1,00-1,06$ & 0,048 & & & \\
\hline Comorbidity & 2,27 & $1,14-4,50$ & 0,019 & & & \\
\hline Oral Hygiene & 0,40 & $0,25-0,64$ & 0,000 & & & \\
\hline Renal Stone History & 44,55 & $14,10-140,78$ & 0,000 & 34,59 & $10,21-117,22$ & 0,000 \\
\hline Urine $\mathrm{pH}$ & 1,97 & $1,05-3,70$ & 0,035 & & & \\
\hline
\end{tabular}

Table 3.

Univariate and multivariate analyses of patient information. tional disease, oral hygiene level, stone story, and urinary $\mathrm{pH}$ values. In the multivariate model, significant and independent $(p<0.05)$ efficacy was observed in the age of the patients, the percentage of tooth stones, and the stone story, suggesting stone formation (Table 3).

\section{Discussion}

Most of the stones in our bodies can be defined as pathological calcifications. In the literature, there are thousands of articles associated with calcifications occurring in the body, however, this amount is less than the number of articles attempting to explain the relationship between them. Stones occurring in different organs resemble their structure suggesting a metabolic disorder that underlies the process of their formation $(6,7)$. The components of stone formation are similar in many organs (8). Davidovich E. et al. evaluated the correlation between dental calculus and disturbed mineral metabolism in pediatric patients with chronic kidney disease. They suggested that there is a possible association between the severity of renal dysfunction in young patients and the formation of dental calculus as an additional manifestation of disturbed calcium-phosphate homeostasis. The combination of several components in saliva including calcium, phosphate, uric acid, and magnesium plays an important role in this process (9). Looking at the minerals that make up kidney stones, we see the same accumulation in the kidneys and teeth, suggesting similar mechanisms of stone formation (10). 
Some researchers have found that nephrolithiasis is also accepted as a predisposing factor to pulpal calcification; however, some others could not find any correlation between the presence of pulp stones and kidney stones $(11,12)$. Some researchers have proposed that some of the minerals found in saliva cause the formation of stones in the salivary glands. Salivary sialoliths are predominantly composed of crystals comprising calcium and phosphorous, with small amounts of magnesium, sodium, chloride, silicon, iron, and potassium (13). Rakesh $N$. et al. found that, when comparing sialoliths and nephroliths, they found a high degree of elemental similarity between them. Thus, they alleged that prescription drugs used for renal stones may be of some use in the conservative management of sialoliths (14). Shaimaa et al. evaluated idiopathic calcium renal stones and their relationship to dental calculi. They reported significant correlations between dental calculus accumulations and calcium renal stone formation, and they offered oral health preventive programs for those patients (15). Grases et al. found that salivary calcium concentrations of patients with hydroxyapatite calculi were significantly higher than that found in the saliva of healthy in their study. Therefore, their results were practically identical to those found in the hydroxyapatite renal calculi (non-infective phosphate renal calculi) (16, 17). We assessed patients based on oral hygiene, renal stone history, teeth brushing habits, dental calculus recurrence, and comorbidities. The dental calculus percentage was significantly higher in patients with kidney stones. This similarity can be seen because of the materials that make up the stone structure tend to accumulate in the body. This may be an indication that kidney stones can form in patients with a high dental calculus density. This condition, which is not related to the recurrence frequency of the dental calculi, is also directly related to oral hygiene status.

\section{Conclusions}

There are similar features in terms of the formation of stones in the body and the minerals they contain.

The height of dental calculi observed in the mouth is significant and stimulating in terms of kidney stones in patients. During physical examinations, the formation of a visible stone, such as a dental calculus, can be a predictor of stones such as kidney stones, and this relationship should be further investigated.

\section{REFERENCES}

1. Turesky SS. What is the role of dental calculus in the etiology and progression of periodontal disease? J Periodontol. 1970; 41:285-6.

2. Beiswanger BB, Segreto VA, Mallatt ME, Pfeiffer HJ. The prevalence and incidence of dental calculus in adults. J Clin Dent. 1989; 1:55-8.

3. Jin Y, Yip HK. Supragingival calculus: formation and control. Crit Rev Oral Biol Med. 2002; 13:426-41.

4. Coe FL, Worcester EM, Evan AP. Idiopathic hypercalciuria and formation of calcium renal stones. Nat Rev Nephrol. 2016; 12:519-33.
5. Worcester EM, Coe FL. Clinical practice. Calcium kidney stones. N Engl J Med. 2010; 363:954-63.

6. Avogaro A, Fadini GP Mechanisms of ectopic calcification: implications for diabetic vasculopathy. Cardiovasc Diagn Ther. 2015; 5:343-52.

7. Valenzuela A, Chung L. Calcinosis: pathophysiology and management. Curr Opin Rheumatol. 2015; 27:542-8.

8. Aguilar-Ruiz J, Arrabal-Polo MA, Sierra M, Arrabal-Martin M. Application of mineralogical techniques in the study of human lithiasis. Ultrastruct Pathol. 2012; 36:367-76.

9. Davidovich E, Davidovits M, Peretz B, et al. The correlation between dental calculus and disturbed mineral metabolism in paediatric patients with chronic kidney disease. Nephrol Dial Transplant. 2009; 24:2439-45.

10. Khan SR, Pearle MS, Robertson WG, et al. Kidney stones. Nat Rev Dis Primers. 2016; 2:16008.

11. Nayak M, Kumar J, Prasad LK. A radiographic correlation between systemic disorders and pulp stones. Indian J Dent Res. 2010; 21:369-73

12. Tarim Ertas E, Inci M, Demirtas A, et al. A Radiographic Correlation between Renal and Pulp Stones West Indian Med J. 2014; 63:620

13. Hiraide F, Nomura Y. The fine surface structure composition of salivary calculi. Laryngoscope 1980; 90:152.

14. Rakesh N, Bhoomareddy Kantharaj YD, Agarwal M, Agarwal K. Ultrastructural and elemental analysis of sialoliths and their comparison with nephroliths. J Investig Clin Dent. 2014; 5:32-7.

15- Shaimaa KY, Mohammed SA. Dental calculus in relation to idiopathic calcium renal stone J Bagh Coll Dentistry. 2012; 24(sp. Issue 1):140-145.

16. Grases F, Santiago C, Simonet B. Sialolithiasis: mechanisim of calculi formation and etiologic factors. Clin Chim Acta. 2003; 334:131-6.

17. Grases F, Sohnel O, Villacampa AI, March JG. Phosphates precipitating from artificial urine and fine structure of phosphate renal calculi. Clin Chim Acta. 1996; 244:45-67.

\section{Correspondence}

Bulent Kati, MD

bulentkati@harran.edu.tr

Eyyup Sabri Pelit, MD

dreyyupsabri@hotmail.com

Ismail Yagmur, MD

dr_iyagmur@hotmail.com

Halil Çiftçi, MD

halilciftci63@hotmail.com

Harran University, Favulty of Medicine Hospital, Urology, 63340

Sanliurfa, Turkey

Ergin Kalkan, MD

erginkalkan@hotmail.com

Assistant Professor of Urology

Cagri Dental Hospital, Dental Clinic, 23100, Elazig, Turkey 